\title{
La campaña virtual en Twitter: análisis de las cuentas de Rajoy y de Rubalcaba en las elecciones generales de 2011
}

\author{
Carmela García ORTEGA \\ Universidad San Jorge \\ cgarcia@usj.es \\ Ricardo Zugasti Azagra \\ Universidad San Jorge \\ rzugasti@usj.es
}

\begin{abstract}
Resumen:
Twitter es la red social en Internet que mayor interés despierta para la comunicación política. Este artículo pretende llenar parcialmente la escasez de investigaciones sobre su empleo electoral en España con un estudio de las cuentas de los dos principales candidatos a la presidencia del Gobierno en 2011. Para ello se analizó cuantitativamente el contenido de cada uno de los 2.319 tweets publicados en ambas cuentas durante la campaña electoral con el objeto de conocer los temas de campaña, así como diversas características de los tweets en relación con su nivel de interactividad.
\end{abstract}

Palabras clave: Twitter; comunicación política; campañas electorales.

\section{The virtual campaign: Analysis of the Twitter accounts of Rajoy and Rubalcaba in the 2011 Spanish general elections}

\begin{abstract}
:
Twitter is an online social networking service which has generated a special interest regarding political communication. This piece of research aims to offer one of the first studies about the use of Twitter in a Spanish electoral context through an analysis of the two main candidates' Twitter accounts during the 2011 general elections campaign. To that end a quantitative content analysis was applied to every tweets posted in both accounts (2,319 in total). The analysis focuses on identifying the campaign issues as well as knowing the level of interactivity.
\end{abstract}

Key Words: Twitter; political communication; electoral campaigns.

\section{Referencia normalizada:}

García Ortega C. y Zugasti Azagra, R. (2014): La campaña virtual en twitter: análisis de las cuentas de Rajoy y de Rubalcaba en las elecciones generales de 2011. Historia y Comunicación Social. Vol. 19. Núm. Especial Febrero. Págs. 299-311.

Sumario: 1. Introducción: Twitter en la comunicación política. 2. Metodología. 3. Presentación de resultados. 3.1. Rajoy y Rubalcaba, ¿dos candidatos dialogantes en Twitter? 3.2. ¿De qué hablan Rajoy y Rubalcaba en Twitter? 4. Conclusiones. 5. Bibliografía. 


\section{Introducción: Twitter en la comunicación política}

Internet está modificando buena parte de los paradigmas comunicativos tradicionales en nuestras sociedades, en especial gracias a la extensión de la web 2.0 con el comienzo del nuevo milenio (Stieglitz y Dang-Xuan, 2012: 1-2). La política no ha quedado al margen y ha ido incorporando en los últimos años la Red como instrumento al servicio de sus estrategias comunicativas (Benkler, 2006; Farrell y Drezner, 2008; Caldevilla, 2009).

Las páginas web fueron, a mediados de la década de 1990, el primer uso de Internet en este ámbito por parte de partidos y candidatos (Dader, 2009: 45). A partir más o menos de 2000 se sumaron los blogs. Las redes sociales irrumpieron con fuerza desde 2002 y muy pronto alcanzaron audiencias masivas: Fotolog (2002), LinkedIn (2003), MySpace (2003), Last.FM (2003), Hi5 (2003), Orkut (2004), Flickr (2004), Facebook (2004), YouTube (2005), Bebo (2005), Ning (2005) y Twitter (2006) (Pérez-Latre, Portilla y Sánchez Blanco, 2011: 64-65). El principal hito en el empleo de las redes sociales en política, propulsor de su uso en otros países, fue la campaña electoral de Obama en 2008, que le llevó hasta la presidencia de los Estados Unidos (Wattal, Schuff, Mandviwalla y Williams, 2010; Cogburn y Spinoza-Vásquez, 2011).

En la actualidad, Twitter es la red social que más relieve ha conseguido entre políticos y periodistas (Rodríguez y Ureña, 2012: 90; Noguera Vivo, 2013: 94). Existen en el mundo más de 380 millones de cuentas de Twitter que difunden cientos de millones de mensajes diariamente (Semiocast, 2012). En 2011, el 13,82\% de la población española tenía una cuenta (Figueroa Daza, González Hernández y Núñez de Prado, 2013: 493) y el 32\% de los internautas españoles usaba esta red (Observatorio de Redes Sociales, 2012). En términos de actividad de las cuentas, España ocupa además el tercer lugar mundial, sólo superada por Holanda y Japón. El 29\% de las cuentas de Twitter ubicadas en España emitieron al menos un mensaje público entre el 1 de septiembre y el 30 de noviembre de 2011 (Semiocast, 2012).

A finales de 2011, coincidiendo con la campaña electoral, el 61\% de los usuarios españoles de Twitter eran hombres y el 39\%, mujeres. Son mayoritarios los usuarios jóvenes, ya que la edad media estaba en torno a los 28 años. El auge de este sistema de microblogging es reciente: el 55\% de los twitteros españoles en 2011 abrieron sus cuentas ese mismo año (Observatorio de Redes Sociales, 2012). En la esfera política, una encuesta del Centro de Investigaciones Sociológicas realizada tras las elecciones generales de 2011 reveló que el $15,2 \%$ de los encuestados tenía una cuenta de Twitter y que el 17,6\% había seguido información sobre la campaña o las elecciones a través de alguna red social (CIS, 2011-2012).

En paralelo con su expansión como herramienta de comunicación política, ha comenzado la investigación académica, todavía incipiente, sobre este empleo concreto de Twitter. Pocos autores se han aventurado a comprobar la influencia o el efecto de Twitter en el establecimiento de la agenda de temas, el cambio de actitudes políticas de los ciudadanos o en la intención de voto; y los que lo han hecho, como 
Gaffney (2010) o Hong y Nadler (2011), no han sido capaces de encontrar evidencias sólidas de su influencia. La mayoría de trabajos se ha centrado en conocer qué uso se le da, cómo se produce la interactividad y cómo es el contenido de los mensajes publicados (Stieglitz y Dang-Xuan, 2012: 3-4).

Además, la investigación sobre Twitter en relación con la política ha girado mayoritariamente sobre dos grandes ejes. Por un lado, ha abordado cómo es el uso que de esta red hacen los políticos a través, por ejemplo, de estudios como los de Golbeck, Grimes y Rogers (2010), y Lassen y Brown (2011), centrados en los congresistas estadounidenses. Por otro lado, ha tratado de conocer el empleo político de Twitter por parte de los ciudadanos. En este sentido, destacan trabajos como el de Yardi y Boyd (2010), enfocado a conocer con quiénes interactúan los twitteros cuando se trata de política; o el de Tumasjan, Sprenger, Sandner y Welpe (2011), que analizó las menciones a partidos y a políticos alemanes en un contexto electoral.

Se echan de menos, sin embargo, investigaciones que arrojen algo de luz sobre el uso de Twitter en la comunicación política en España. Este es el principal objetivo que se propone este artículo, a través del análisis de dos casos de uso de Twitter en un contexto electoral: el que hicieron Mariano Rajoy (@marianorajoy) y Alfredo Pérez Rubalcaba (@conRubalcaba) desde sus cuentas oficiales durante la campaña de las elecciones generales de noviembre de 2011. Ambos eran candidatos, respectivamente, del Partido Popular y del Partido Socialista a la presidencia del Gobierno.

Además, fue precisamente la campaña para estas elecciones la que consagró plenamente Twitter como un instrumento comunicativo en España. Sirva como ejemplo que Alfredo Pérez Rubalcaba abrió su perfil el 8 de julio de 2011, un día después de que asumiera su candidatura en el Comité Federal del PSOE. Mariano Rajoy lo hizo algo más tarde, el 15 de septiembre, con el cercano objetivo de las elecciones generales. Eran principalmente los equipos de campaña de ambos candidatos los gestores de las cuentas.

\section{Metodología}

La metodología empleada en esta investigación ha sido el análisis de contenido. Para ello, se diseñó un código que se aplicó a cada uno de los tweets publicados por Mariano Rajoy y Alfredo Pérez Rubalcaba desde el día que se inició la campaña electoral hasta el día de las votaciones. Las categorías del código contemplaban, entre otros aspectos, los temas principal y secundario de los mensajes, así como el uso de etiquetas y enlaces, la inclusión de menciones y a quién se realizaban, o la procedencia de los retweets. En total se analizaron 2.319 tweets: 927 publicados en la cuenta de Rajoy y 1.392, en la de Rubalcaba.

La selección de variables en el código buscaba comprobar empíricamente si el empleo de Twitter por parte de ambos políticos tuvo en cuenta la característica que mejor diferencia el empleo de la web 2.0: el diálogo frente a la comunicación unidi- 
reccional. En este sentido, algunos autores como Castells han evidenciado que los partidos políticos han usado Internet como si se tratara de "un tablón de anuncios digital" (2009: 12). Se trataba por lo tanto de identificar, entre otros factores, el grado de interacción de@marianorajoy y de @conRubalcaba. Asimismo, interesaba saber si ambas cuentas incluían temas más propios de la agenda de la ciudadanía que de la agenda de los candidatos. Por ello se analizaron los temas de los tweets, así como la importancia de cada uno de estos issues de la campaña virtual a través de Twitter.

\section{Presentación de resultados}

El análisis detallado de los 2.319 tweets publicados por Mariano Rajoy y Alfredo Pérez Rubalcaba entre el 4 y el 20 de noviembre nos permitirá describir el uso que los dos candidatos hicieron de esta red social durante la campaña electoral, así como detectar similitudes y diferencias entre ambos. En primer lugar atenderemos a algunas cuestiones que nos ayudarán a determinar si los dos políticos aprovecharon el potencial dialógico de Twitter y después analizaremos la temática sobre la que versaron los mensajes de ambos candidatos.

\subsection{Rajoy y Rubalcaba, ¿Dos candidatos dialogantes en twitter?}

Una de las principales ventajas de Twitter, tal y como se ha comentado, es que permite al político establecer pequeños diálogos con otros usuarios. Es decir, ofrece la posibilidad de crear situaciones de comunicación bidireccionales donde antes resultaba prácticamente imposible. Además, el candidato ya no es siempre el que toma la iniciativa en la relación con otros sujetos sino que, a veces, tiene que reaccionar ante las preguntas, peticiones, comentarios o críticas que otras personas le plantean. Esa capacidad para responder, para interactuar, puede resultar muy positiva si se gestiona adecuadamente, es decir, si los políticos entienden que "Twitter no es sólo hablar, sino también escuchar, responder y debatir" (Rodríguez Andrés y Ureña, 2012: 94).

Rajoy y Rubalcaba realizaron menciones a otros usuarios de Twitter en un 68\% de los mensajes analizados en esta investigación, lo que evidencia una notable capacidad dialógica. La mayoría de las menciones (tabla 1) tuvo como destinatario a un ciudadano $(69,6 \%)$. Sin embargo, el comportamiento de los dos candidatos a la presidencia del Gobierno presentó algunas diferencias.

La capacidad para interactuar con la ciudadanía fue mayor en el caso del político popular que en el del socialista: Rajoy realizó un 77,6\% de menciones, mientras que Rubalcaba se quedó en el $62,8 \%$. Sin embargo, este último tuvo una mayor relación con la clase política: hizo referencia a algún político de su partido en un 17,7\% de sus mensajes, frente al 9,8\% de Rajoy, y aludió a los políticos de la oposición en un $6,6 \%$ de los casos, por delante del 1,1\% registrado por el vencedor de las elecciones. 
Llama la atención que sean los periodistas y medios de comunicación los menos mencionados en los tweets de los dos candidatos (2,8\%). Rubalcaba los citó en un $3,2 \%$ de los casos y Rajoy en un $2,3 \%$. A la luz de estos datos, podemos afirmar que Twitter no se añadió a los instrumentos de comunicación habitualmente empleados entre políticos y periodistas.

Tabla 1. Menciones por candidato (en porcentaje)

\begin{tabular}{|c|c|c|c|}
\hline & Rajoy & Rubalcaba & Total \\
\hline Políticos de su partido & 9,8 & 17,7 & 14 \\
\hline Políticos de la oposición & 1,1 & 6,6 & 4,1 \\
\hline Periodistas/medios & 2,3 & 3,2 & 2,8 \\
\hline Ciudadanos & 77,6 & 62,8 & 69,6 \\
\hline Otros & 5,2 & 3,1 & 4,1 \\
\hline Varias de las anteriores & 4 & 6,6 & 5,4 \\
\hline Total & $\mathbf{1 0 0}$ & $\mathbf{1 0 0}$ & $\mathbf{1 0 0}$ \\
\hline
\end{tabular}

El retweet no fue muy utilizado ni por Rajoy ni por Rubalcaba. De todos los mensajes analizados para este artículo, solo un 19,7\% fue retweet. Los dos políticos tendieron a retweetear, sobre todo, mensajes publicados en las cuentas de sus partidos $(69,6 \%)$. No obstante, Alfredo Pérez Rubalcaba $(71,9 \%)$ destacó sobre Mariano Rajoy $(55,4 \%)$ en este sentido. Por el contrario, el candidato del PP adelantó considerablemente al del PSOE si atendemos al porcentaje de retweets tanto de periodistas y medios de comunicación $(18,5 \%$ frente a $5,4 \%)$ como de otros políticos $(6,2 \%$ frente a 3,3\%). Los retweets de ciudadanos presentan unos porcentajes muy similares en ambos candidatos, en torno al 19,5\% (tabla 2)

Tabla 2. Retweet por candidato (en porcentaje)

\begin{tabular}{|c|c|c|c|}
\hline & Rajoy & Rubalcaba & Total \\
\hline RT cuenta de partido & 55,4 & 71,9 & 69,6 \\
\hline RT otro político & 6,2 & 3,3 & 3,7 \\
\hline RT ciudadano & 20 & 19,4 & 19,5 \\
\hline RT periodista/medio & 18,4 & 5,4 & 7,2 \\
\hline Total & $\mathbf{1 0 0}$ & $\mathbf{1 0 0}$ & $\mathbf{1 0 0}$ \\
\hline
\end{tabular}

El empleo de etiquetas (hashtags) por parte de los dos candidatos resultó bastante similar. Rajoy las incluyó en un $41,9 \%$ de sus mensajes, mientras que Rubalcaba se quedó en el 36,3\% (tabla 3). Sin embargo, en términos absolutos, el político socialista utilizó más etiquetas que el popular (630 frente a 477), ya que buena parte de sus tweets incluían varios hashtags.

El análisis de las etiquetas más empleadas por ambos candidatos muestra que estas funcionaron más bien como eslóganes de campaña que se añadían a buena parte de los tweets, y no tanto como indicadores de contenido. Mariano Rajoy empleó 44 
etiquetas diferentes, pero hubo una que destacó sobre las demás, \#progamaPP, pues se empleó en un $43,6 \%$ de los casos. Por detrás, nos encontramos con \#sumatealcambio (14,2\%), \#2diasparaelcambio (6,7\%) y \#rajoygana (5,9\%). El resto de hashtags presentó una frecuencia de aparición inferior al 5\%.

Rubalcaba, por su parte, hizo uso de 109 etiquetas distintas. Las más mencionadas tenían una cierta similitud: \#yorespondo $(12,8 \%)$ y \#rajoynorespondealfredonoseesconde $(8,9 \%)$. Por detrás se situaron \#peleaporloquequieres $(8,7 \%)$ y \#votaPSOE (7,5\%). La frecuencia de aparición del resto de hashtags no superó el 4\%.

Tabla 3. Hashtag por candidato (en porcentaje)

\begin{tabular}{|c|c|c|c|}
\hline & Rajoy & Rubalcaba & Total \\
\hline Sí & 41,9 & 36,3 & 38,5 \\
\hline No & 58,1 & 63,7 & 61,5 \\
\hline Total & $\mathbf{1 0 0}$ & $\mathbf{1 0 0}$ & $\mathbf{1 0 0}$ \\
\hline
\end{tabular}

Entre los recursos que ofrece Twitter se encuentra la posibilidad de incluir enlaces, algo que hicieron los dos candidatos en un $30,7 \%$ de sus mensajes. Los enlaces a páginas web destacaron claramente sobre el resto, pues se realizaron en un $69,3 \%$ de las ocasiones, muy por delante de las fotografías $(17,8 \%)$, los vídeos $(10,8 \%)$ o las localizaciones $(0,3 \%)$.

Si atendemos a cada uno de los candidatos por separado, observamos dos diferencias en el uso de este recurso (tabla 4). Rajoy superó a Rubalcaba en enlaces a sitios web $(79 \%$ frente a $58,8 \%)$, mientras que el candidato socialista adelanto al popular en vídeos (19\% frente a 3,2\%).

Tabla 4. Enlaces por candidato (en porcentaje)

\begin{tabular}{|c|c|c|c|}
\hline & Rajoy & Rubalcaba & Total \\
\hline$W e b$ & 79 & 58,8 & 69,3 \\
\hline Foto & 17,5 & 18,1 & 17,8 \\
\hline Vídeo & 3,2 & 19 & 10,8 \\
\hline Localización & 0,3 & 0,3 & 0,3 \\
\hline Otros & 0 & 1,2 & 0,6 \\
\hline Varias de las anteriores & 0 & 2,6 & 1,2 \\
\hline Total & $\mathbf{1 0 0}$ & $\mathbf{1 0 0}$ & $\mathbf{1 0 0}$ \\
\hline
\end{tabular}

3.2. ¿De qué hablan Rajoy y Rubalcaba en twitter?

Nos detendremos ahora en el análisis temático de los tweets de Mariano Rajoy y de Alfredo Pérez Rubalcaba. Conocer de qué asuntos hablaron cada uno de ellos y con qué frecuencia lo hicieron nos permitirá saber, como mencionábamos anteriormente, 
si predominaron los asuntos propios de la agenda de los candidatos o si se dio cabida a otros.

Un tema destacó sobre los demás en las cuentas@marianorajoy y @conRubalcaba: la organización de la campaña (tabla 5). Prácticamente la mitad de los tweets publicados por ambos políticos se centró en esta cuestión (47,9\%), si bien Rubalcaba se ocupó de este asunto $(54,5 \%)$ bastante más que Rajoy (37,9\%). Este dato demuestra que los candidatos tendieron a utilizar Twitter a modo de tablón de anuncios en el que daban a conocer a sus seguidores las actividades de su agenda de campaña. Algunas de las etiquetas empleadas por los dos políticos hacían alusión a su presencia en distintos actos de campaña a lo largo y ancho del territorio español. Esto fue más habitual en el caso del líder socialista, en cuya cuenta encontramos varios hashtags similares: \#andaluciaconrbcb, \#balearesambrbcb, \#burgosconrubalcaba, \#huelvaconrbcb... Además, dos etiquetas hacían referencia al carácter itinerante de la campaña: \#mitinesenruta, que se empleó en un 2,9\% de los casos, y \#busPSOE, que fue utilizada en un $1,3 \%$. Aunque en menor medida, en la cuenta de Rajoy también se encontraron algunas etiquetas referidas al lugar donde se encontraba el candidato popular en ese momento: \#rajoyenoviedo, \#plazadetorosdevalencia, \#extremaduraconrajoy...

Así pues, Rajoy y Rubalcaba tendieron a emplear Twitter para hablar, principalmente, sobre la propia campaña electoral. En este sentido, se observa una coincidencia con el modo de trabajar de los medios de comunicación, pues tal y como han puesto de manifiesto algunos trabajos, el asunto más mencionado por los periodistas cuando cubren campañas es, precisamente, el modo en el que ésta se ha diseñado y se está desarrollando (García Ortega y Verón Lassa, 2012).

El segundo tema más presente en las cuentas de los dos candidatos a la presidencia del Gobierno fue derechos sociales $(12,7 \%)$. Ambos políticos prestaron una atención similar a este asunto aunque Rajoy $(13,9 \%)$ adelantó ligeramente a Rubalcaba $(11,9 \%)$.

La crisis económica (12\%) fue el tercer asunto más mencionado y, en esta ocasión, los dos candidatos le dedicaron el mismo porcentaje de tweets. Otros asuntos económicos, al margen de la crisis, ocuparon el 9,4\% de los mensajes analizados. La diferencia entre los dos políticos en este caso fue mayor. El candidato popular dedicó un $16,5 \%$ de tweets a este tema, bastante por delante del $4,8 \%$ registrado por el líder socialista.

Si consideramos de manera conjunta los dos temas económicos, éstos aparecieron en más de un quinto de los tweets $(21,4 \%)$. Esa presencia fue mayor en el caso de Rajoy (28,5\%) que en el de Rubalcaba (16,8\%).

El resto de las cuestiones contempladas en esta investigación presentó una frecuencia de aparición menor, por debajo del 2,5\%. Resulta llamativo que temas considerados importantes por los ciudadanos, como por ejemplo la inmigración (CIS, 2011), apenas fueran mencionados. Rajoy se refirió a esta cuestión en un 1,4\% de los mensajes y Rubalcaba no lo hizo en ningún caso. También son muy escasas las 
referencias al 15M: Rajoy no lo mencionó y Rubalcaba lo hizo, solamente, en el 0,1\% de sus tweets, algo sorprendente pues fueron considerables los guiños del político socialista a los integrantes de este movimiento durante toda la campaña. Ninguno de los dos candidatos se ocupó de la polémica en torno a la participación de la izquierda abertzale en las elecciones. Rajoy no mencionó datos de encuetas preelectorales y Rubalcaba, al que la mayoría de esos sondeos daban como perdedor, lo hizo en un $0,5 \%$ de sus mensajes.

Tabla 5. Tema principal por candidato (en porcentaje)

\begin{tabular}{|c|c|c|c|}
\hline & Rajoy & Rubalcaba & Total \\
\hline Organización campaña & 37,9 & 54,5 & 47,9 \\
\hline Derechos sociales & 13,9 & 11,9 & 12,7 \\
\hline Crisis económica & 12 & 12 & 12 \\
\hline Otros economía & 16,5 & 4,8 & 9,4 \\
\hline Participación/abstención & 1,6 & 2,6 & 2,2 \\
\hline Ley electoral & 1,7 & 1,3 & 1,5 \\
\hline Estado autonómico & 1,4 & 0,6 & 1 \\
\hline Corrupción/escándalos políticos & 0,5 & 1 & 0,8 \\
\hline Mujer & 1 & 0,6 & 0,8 \\
\hline Inmigración & 1,4 & 0 & 0,6 \\
\hline Energía & 0,5 & 0,6 & 0,6 \\
\hline ETA/terrorismo & 0,8 & 0,2 & 0,4 \\
\hline Infraestructuras & 0,4 & 0,4 & 0,4 \\
\hline Vivienda & 0,6 & 0,2 & 0,4 \\
\hline Encuestas & 0 & 0,5 & 0,3 \\
\hline $15 \mathrm{M}$ & 0 & 0,1 & 0,1 \\
\hline Otros & 9,8 & 8,7 & 8,9 \\
\hline Total & $\mathbf{1 0 0}$ & $\mathbf{1 0 0}$ & $\mathbf{1 0 0}$ \\
\hline & & & \\
\hline & & & 0,3 \\
\hline
\end{tabular}

Junto al tema principal, en un $15,9 \%$ de los tweets encontramos, además, un asunto secundario. La organización de campaña volvió a ser, también ahora, lo más mencionado (25,5\%), si bien en esta ocasión Rajoy $(31,3 \%)$ adelantó a Rubalcaba $(16,3 \%)$. Las cuestiones económicas fueron el tema secundario en un $41 \%$ de los tweets analizados. De la crisis habló más el político popular (24,7\%) que el socialista (22\%), al contrario de lo que ocurrió con otros asuntos económicos $(15,4 \%$ para Rajoy y 20,6\% para Rubalcaba).

Derechos sociales fue el cuarto tema más mencionado $(16,8 \%)$ y, esta vez, Rubalcaba $(18,4 \%)$ se situó por delante de Rajoy $(15,4 \%)$. Por detrás, a cierta distancia, nos encontramos con un 4,3\% de tweets relativos a la organización territorial de España. Fue este un asunto al que Rubalcaba dio más importancia que Rajoy, pues el primero 
se refirió al estado autonómico en casi en un $10 \%$ de sus mensajes, mientras que el segundo no alcanzó el 1\%. Algo similar se observa si nos fijamos en el tema relativo a la participación/abstención en las elecciones, pues estuvo más presente en la cuenta de Rubalcaba $(6,4 \%)$ que en la de Rajoy $(2,2 \%)$. La frecuencia de aparición del resto de issues resultó muy baja y, salvo en el caso de la vivienda (1,1\%), estuvo por debajo del $1 \%$. Merece la pena destacar, antes de terminar, que entre los temas secundarios del candidato socialista no se encontraron referencias ni a infraestructuras, ni a energía, ni a inmigración, ni a corrupción y escándalos políticos. Por su parte, el vencedor en las elecciones del $20 \mathrm{~N}$ no hizo ninguna referencia a ETA o al terrorismo (tabla 6).

Tabla 6. Tema secundario por candidato (en porcentaje)

\begin{tabular}{|c|c|c|c|}
\hline & Rajoy & Rubalcaba & Total \\
\hline Organización campaña & 31,3 & 16,3 & 25,5 \\
\hline Crisis económica & 24,7 & 22 & 23,6 \\
\hline Otros economía & 15,4 & 20,6 & 17,4 \\
\hline Derechos sociales & 15,9 & 18,4 & 16,8 \\
\hline Estado autonómico & 0,9 & 9,9 & 4,3 \\
\hline Participación/abstención & 2,2 & 6,4 & 3,8 \\
\hline Vivienda & 1,3 & 0,7 & 1,1 \\
\hline Mujer & 0,9 & 0,7 & 0,8 \\
\hline Ley electoral & 0,4 & 0,7 & 0,5 \\
\hline Infraestructuras & 0,9 & 0 & 0,5 \\
\hline Energía & 0,9 & 0 & 0,5 \\
\hline ETA/terrorismo & 0 & 0,7 & 0,3 \\
\hline Inmigración & 0,4 & 0 & 0,3 \\
\hline Corrupción/escándalos políticos & 0,4 & 0 & 0,3 \\
\hline Otros & 4,4 & 3,6 & 4,3 \\
\hline Total & $\mathbf{1 0 0}$ & $\mathbf{1 0 0}$ & $\mathbf{1 0 0}$ \\
\hline
\end{tabular}

\section{Conclusiones}

Tanto Rajoy como Rubalcaba establecieron un cierto nivel de conversación con otros usuarios a través del uso de menciones. En este sentido, explotaron las posibilidades de la red de microblogging para interactuar, sobre todo, con ciudadanos. Menor fue la interacción con otros políticos, mayoritariamente del propio partido, y muy escasa con periodistas o medios de comunicación, lo que muestra que Twitter no se empleó durante la campaña como vía de diálogo con la prensa. Distinguiendo entre cuentas, destaca que Rubalcaba mencionó más a políticos que su oponente y que este último realizó más menciones a ciudadanos que no eran políticos ni periodistas. 
Si el empleo de las menciones fue notable, menor fue el recurso al retweet por ambos candidatos. Sobresale en este sentido que la mayoría de los retweets de @ marianorajoy y@conRubalcaba lo fue, respectivamente, de mensajes procedentes de las cuentas oficiales del Partido Popular y del Partido Socialista. Destacó el líder socialista al retweetear mensajes procedentes de su propia formación. Por su parte, Rajoy utilizó bastante el retweet para difundir mensajes procedentes de cuentas de periodistas y de medios de comunicación, lo que matiza parcialmente el resultado mostrado anteriormente acerca de las referencias a la prensa por parte de los candidatos.

En cuanto al uso de etiquetas, Rubalcaba incluyó más en sus tweets y con menor frecuencia, mientras que Rajoy empleó menos y con más frecuencia, lo que evitó la dispersión. En todo caso, los hashtags más recurrentes funcionaron más bien como la adaptación de eslóganes de campaña a Twitter, y no tanto como indicadores del contenido de los mensajes, lo que apunta a su empleo dentro de los cánones tradicionales del marketing electoral.

Ambas cuentas hicieron un uso habitual de los enlaces. Tanto Rajoy como Rubalcaba primaron los enlaces a una página web, lo que constituye un intento por aportar información más abundante que rompa el corsé de los 140 caracteres. Distinguiendo entre candidatos, fue el popular quien más enlazó a webs en sus mensajes, mientras que el socialista destacó por los enlaces a vídeos, algo que apunta a un mayor interés por vincular Twitter con los productos audiovisuales de campaña.

Por lo que respecta a los temas de los tweets, Rajoy y Rubalcaba usaron esta red social como un tablón de anuncios o altavoz de sus actos electorales. En este sentido, Twitter se convierte en algo autorreferencial, es decir, en una herramienta para hablar de los propios actos de la campaña presencial tradicional. En definitiva, no se explotan sus posibilidades para llevar a cabo una campaña virtual con desarrollo propio en Internet.

Siguiendo con los temas, ambas cuentas prestaron especial atención a los asuntos relacionados con la crisis económica, lo que indica en este caso una coincidencia entre la agenda política y la agenda ciudadana. Sin embargo, esta coincidencia es parcial, pues el Twitter de los candidatos apenas habla de temas como la inmigración, la corrupción y los escándalos políticos, o el movimiento $15 \mathrm{M}$, asunto este último que prácticamente monopolizó la recta final de la campaña de las elecciones autonómicas y municipales de mayo de 2011.

\section{Bibliografía}

BENKLER, Y. (2006). The wealth of networks: How social production transforms markets and freedom. New Haven: Yale University Press. 
CALDEVILLA DOMÍNGUEZ, D. (2009). "Democracia 2.0: La política se introduce en las redes sociales". En: Pensar la Publicidad. Revista Internacional de Investigaciones Publicitarias, $\mathrm{n}^{\circ}$ 2, vol. 3, p. 31-48.

CASTELLS, M. (2010). "La democràcia en l'era d'Internet". En: VIA. Valors, idees, actituds: revista del Centre d'Estudis Jordi Pujol, nº 12, p. 7-13.

CENTRO DE INVESTIGACIONES SOCIOLÓGICAS (2011): Barómetro de noviembre, estudio número 2.917. Disponible en http://datos.cis.es/pdf/ Es2917mar_A.pdf. Consultado el 9 de junio de 2012.

CENTRO DE INVESTIGACIONES SOCIOLÓGICAS (2011-2012). "Postelectoral elecciones generales 2011, estudio número 2.920". Disponible en http://datos.cis. es/pdf/Es2920mar_A.pdf [9-06-2013].

COGBURN, D. L., y SPINOZA-VÁSQUEZ, F. K. (2011). "From Networked Nominee to Networked Nation: Examining the Impact of Web 2.0 and Social Media on Political Participation and Civil Engagement in the 2008 Obama Campaign". En: Journal of Political Marketing, vol. 10, p. 189-213.

DADER, J. L. (2009). "Ciberpolítica en los websites de partidos políticos: la experiencia de las elecciones de 2008 en España ante las tendencias transnacionales". En: Revista de Sociología y Política, $\mathrm{n}^{\circ}$ 34, p. 45-62.

FARRELL, H., y DREZNER, D. (2008). "The power and politics of blogs". En: Public Choice, $\mathrm{n}^{\mathrm{o}} 134$ (1), p. 15-30.

FIGUEROA DAZA, J., GONZÁLEZ HERNÁNDEZ, E., y NUÑEZ DE PRADO, S. (2013). "Las redes sociales como contexto para la gestión de la marca del candidato en las elecciones generales españolas 2011". En CRESPO, I. Partidos, medios y electores en procesos de cambio. Las elecciones generales españolas de 2011. Valencia: Tirant Humanidades, p. 485-510.

GAFFNEY, D. (2010). "\#iranElection: quantifying online activism". En Proceedings of the WebSci10: Extending the Frontiers of Society On-Line, Raleigh, p. 1-8.

GARCÍA ORTEGA, C., y VERÓN LASSA, J. J. (2012). "La campaña de las municipales y autonómicas de 2011 en Aragón desde la prensa diaria". En CASERO-RIPOLLÉS, A. (ed.). Periodismo político en España: concepciones, tensiones y elecciones. La Laguna: Sociedad Latina de Comunicación Social, Cuadernos Artesanos de Latina, ${ }^{\circ}$ 33, p. 133-148.

GOLBECK, J., GRIMES, J. M., y ROGERS, A. (2010). "Twitter use by the U.S. Congress". En: Journal of the American Society for Information Science and Technology, $\mathrm{n}^{\circ} 61$ (8), p. 1612-1621.

HONG, S., y NADLER, D. (2011). "Does the Early Bird Move the Polls? The use of the social media tool 'Twitter' by U.S. politicians and its impact on public opinion”. En Proceedings of the 12th Annual International Digital Government Research Conference: Digital Government Innovation in Challenging Times, Nueva York: ACM, p. 182-186.

LASSEN, D. S., y BROWN, A. R. (2011). “Twitter: The electoral connection?”. En: Social Sciences Computer Review, no 29 (4), p. 419-436. 
NOGUERA VIVO, J. M. (2013). "How open are journalists on Twitter? Trends towards the end-user journalism". En: Comunicación y Sociedad, n 1, vol. 26, p. 93-114.

OBSERVATORIO DE REDES SOCIALES (2012): "IV Oleada". Disponible en http://www.slideshare.net/TCAnalysis/4-oleada-observatorio-de-redes-sociales [15-06-2013].

PÉREZ-LATRE, F. J., PORTILLA, I., y SÁNCHEZ BLANCO, C. (2011). "Social Networks, Media and Audiences: A Literature Review". En: Comunicación y Sociedad, $\mathrm{n}^{\circ} 1$, vol. 24, p. 63-74.

RODRIGUEZ, R., y URENA, D. (2012). "Diez razones para el uso de Twitter como herramienta en la comunicación política y electoral". En: Comunicación y Pluralismo, $\mathrm{n}^{\circ} 5$, p. 89-116.

SEMIOCAST (2012): "Brazil becomes 2nd country on Twitter, Japan 3rd, Netherlands most active country". Disponible en https://semiocast.com/publications/2012_01_31_Brazil_becomes_2nd_country_on_Twitter_superseds_Japan. [10-06-2013].

STIEGLITZ, S., y DANG-XUAN, L. (2012). "Social media and political communication: a social media analytics framework". En: Social Network Analysis and Mining, vol. 2, p. 1-15.

TUMASJAN, A., SPRENGER, T., SANDNER, P., y WELPE, L, (2011). "Election forecasts with Twitter: how 140 characters reflect the political landscape". En: Social Sciences Computer Review, $\mathrm{n}^{\circ} 29$ (4), p. 402-418.

WATTAL, S., SCHUFF, D., MANDVIWALLA, M., y WILLIAMS, C. (2010). "Web 2.0 and politics: the 2008 U.S. presidential election and an e-politics research agenda". En: MIS Quarterly, no 34 (4), p. 669-688.

YARDI, S., y BOYD, D. (2010). "Dynamic debates: an analysis of group polarization over time on twitter". En: Bulletin of Science, Technology \& Society, nº 20, p.1-8.

\section{Los autores}

Carmela García Ortega es Licenciada en Periodismo y Doctora en Comunicación por la Universidad de Navarra. En la actualidad trabaja como docente e investigadora en la Facultad de Ciencias de la Comunicación de la Universidad San Jorge donde, además, es Vicedecana de Alumnos. Es la investigadora principal del grupo "Medios de comunicación y campañas electorales en Aragón", reconocido como emergente por el Gobierno de Aragón. Sus áreas de investigación son la comunicación política, la historia del periodismo y el estudio de los métodos de investigación en comunicación.

Ricardo Zugasti Azagra es Doctor en Comunicación por la Universidad de Navarra. Es profesor en la Universidad San Jorge de Zaragoza. Asimismo ha sido Visiting Research Fellow en la Universidad de Sheffield (Reino Unido) y profesor visitante en la Universidad del Istmo (Guatemala), en la Burgas Free University (Bulgaria) y en 
el Instituto Politécnico de Lisboa (Portugal). Sus ámbitos de interés investigador son la Historia de la Comunicación y la Comunicación Política. Es miembro del grupo de investigación "Medios de comunicación y campañas electorales", reconocido por el Gobierno de Aragón. 